\title{
Sexually transmitted disease among married \\ Zambian women: the role of male and female sexual behaviour in prevention and management
}

Short report

\author{
C S Morrison, M R Sunkutu, E Musaba, L H Glover
}

Objectives: Few studies have evaluated the relation between male and female sexual behaviour and STD among married African women. The objectives of this study were to identify male and female sexual behaviour associated with female STD, and to explore whether incorporating male and female sexual behaviour and male symptoms can improve algorithms for STD management in married African women.

Methods: 99 married couples with one symptomatic member (58 males, 41 females) attending an STD clinic in Lusaka, Zambia were interviewed separately about sexual and contraceptive behaviour, and had physical examinations. Diagnostic tests for Neisseria gonorrhoeae (GC), Trichomonas vaginalis (TV), and HIV were performed. Bivariate and multivariate odds ratios for the association between sexual behaviour and STD were calculated. Predictive algorithms based on current Zambian guidelines for management of STD in women were created.

Results: Among women at baseline, $10 \%$ were positive for GC, $14 \%$ for TV, $52 \%$ for HIV. Female alcohol use before sex, a male's paying for sex, and a couple's having sex unprotected by condoms or spermicides were associated with female STD. Incorporation of these behaviours along with symptoms of urethral discharge and dysuria among husbands increased the predictive ability of algorithms for management of STD in women.

Conclusions: The addition of male and female sexual behaviour and male STD symptoms to diagnostic algorithms for female STD should be explored in other settings. Both husbands' and wives' behaviour independently predict STD in these women; risk reduction programmes should target both men's and women's sexual behaviour.

(Genitourin Med 1997;73:555-557)

Keywords: sexually transmitted diseases; screening strategy; risk factors

\section{Introduction}

Heterosexual transmission accounts for the vast majority of HIV infections in Africa. Sexually transmitted diseases (STD) including gonorrhoea and trichomoniasis have been identified as cofactors in HIV transmission. ${ }^{1-3}$ While numerous studies have considered risk factors for STD among sex workers, few have examined STD risk factors among married African women.

Studies of African women have identified several behavioural risk factors for HIV infection (for example, a woman having multiple sex partners). Nevertheless, prevalences of such factors among married women are low, ${ }^{34}$ and diagnostic algorithms for female STD incorporating female sexual behaviour have low sensitivity and predictive values. ${ }^{45}$ Correspondingly, some researchers suggest that women's STD risk results primarily from husbands' behaviour and that prevention programmes should target male sexual behaviour. ${ }^{67}$

However, before this study, no other study has jointly assessed the effects of male and female sexual behaviour on STD among married African women nor considered the incorporation of both male and female behaviour into predictive algorithms for female STD.

\section{Methods}

This study was conducted at the National
STD Reference Centre situated at the University Teaching Hospital (UTH) in Lusaka, Zambia. Ninety nine people with symptomatic disease (58 males, 41 females) attending the clinic in 1993-4 who returned with their spouses were enrolled. Sociodemographic, medical history, and sexual and contraceptive behavioural data were collected separately from each partner by questionnaire. Endocervical and urethral swabs for Neisseria gonorrhoeae cultures and smears for wet mount (from the posterior fornix of the vagina and urethra) for Trichomonas vaginalis were taken during genital examinations. Gonorrhoea specimens were inoculated onto ThayerMartin culture medium plates. Oxidase test and Gram stain were used to identify positive gonorrhoea cultures. $T$ vaginalis was identified by the presence of trichomonads on wet mount.

Bivariate associations of risk factors for gonorrhoea and trichomoniasis were examined by calculating crude odds ratios (OR) and 95\% confidence intervals (CI). A restricted set of factors was selected for multivariable analysis using logistic regression. Predictive algorithms were based on current STD management guidelines in Zambia. ${ }^{8}$ Algorithms were created to maximise positive predictive values and likelihood ratios (sensitivity/1 - specificity). Analyses were performed using SAS/STAT (SAS Institute, Cary, NC, USA), and LOGXACT (Cytel Software Corporation, Cambridge, MA, USA). 


\section{Results}

Median age was 35 years for males and 29 years for females; $64 \%$ of males and $41 \%$ of females had at least some secondary education. At admission, $68 \%$ of men were HIV+, $10 \%$ had gonorrhoea, and $29 \%$ reported dysuria or discharge. Among women, $52 \%$ were $\mathrm{HIV}+, 10 \%$ had gonorrhoea, and $14 \%$ had trichomoniasis. Men reported riskier sexual behaviour (previous 3 months) than women including having multiple sex partners ( $40 \% v 6 \%$ ), exchanging money or gifts for sex $(25 \%$ v $5 \%)$, and alcohol use before sex $(37 \% v$ $22 \%)$.

Both a woman's and her husband's behaviour were associated with STD in women. In bivariate analysis, women using alcohol before sex, men having multiple sex partners, and men paying for sex were strongly associated with cervical gonorrhoea. Other variables, including the woman's age and educational level, her number of sex partners in the previous 3 months, and her husband's alcohol use before sex, were not associated with gonorrhoea in women. Multivariable analysis confirmed the association of female alcohol use before sex $(\mathrm{OR}=5 \cdot 2 ; 95 \% \mathrm{CI} 1 \cdot 2-22 \cdot 7)$ and her partner paying for sex $(\mathrm{OR}=6 \cdot 6 ; 95 \% \mathrm{CI}$ 1.5-29.2) with cervical gonorrhoea controlling for condom and spermicide use by the couple. No variable was significantly associated with $T$ vaginalis on microscopy. However, women who used condoms or spermicides inconsistently were more likely to have $T$ vaginalis than consistent users $(\mathrm{OR}=4 \cdot 0 ; 95 \% \mathrm{CI} 0 \cdot 6-\infty)$ controlling for women's alcohol use and men paying for sex.

As expected, a complaint of yellow vaginal discharge $(\mathrm{OR}=19 \cdot 1 ; 95 \%$ CI $2 \cdot 0-180 \cdot 6)$ or yellow or white discharge $(\mathrm{OR}=8 \cdot 2 ; 95 \% \mathrm{CI}$ $1 \cdot 0-67 \cdot 6)$ was highly associated with gonorrhoea. Women with cervical erythema were six times more likely to have gonorrhoea $(\mathrm{OR}=$ $6 \cdot 3 ; 95 \%$ CI $1 \cdot 6-25 \cdot 2$ ). A husband's complaint of urethral discharge was also highly associated with gonorrhoea (OR $=21.0 ; 95 \%$ CI 4.3-103·5). $T$ vaginalis among women was associated with a woman's (OR $=3 \cdot 4 ; 95 \%$ CI $0.9-13 \cdot 0)$ or her husband's (OR $=4 \cdot 3$; $95 \%$ CI 1.3-14.0) complaint of dysuria.

Positive predictive value liklihood ratio, sensitivity, and specificity for clinical signs and symptoms and behavioural variables associated with STD in women

\begin{tabular}{lllll}
\hline & $\begin{array}{l}\text { Positive } \\
\text { predictive } \\
\text { value }\end{array}$ & $\begin{array}{l}\text { Likelihood } \\
\text { ratio }\end{array}$ & Sensitivity & Specificity \\
\hline Predictor variables for Neisseria gonorrhoeae: & & & & \\
Clinics without pelvic exam capacity & & & & \\
(1) Vaginal discharge (any) & $16 \%$ & $1 \cdot 72$ & $90 \%$ & $48 \%$ \\
(2) (1) and female alcohol use & $29 \%$ & 3.52 & $40 \%$ & $89 \%$ \\
(3) (1) and male money for sex & $29 \%$ & $3 \cdot 67$ & $50 \%$ & $86 \%$ \\
(4) (1) and male urethral discharge & $67 \%$ & $17 \cdot 60$ & $40 \%$ & $98 \%$ \\
Clinics with pelvic exam capacity & & & & \\
(1) Yellow vaginal discharge & $31 \%$ & 4.00 & $50 \%$ & $88 \%$ \\
(2) (1) or cervical erythema & $31 \%$ & 3.96 & $90 \%$ & $77 \%$ \\
(3) (2) and female alcohol use & $67 \%$ & $17 \cdot 60$ & $40 \%$ & $98 \%$ \\
(4) (2) and male money for sex & $56 \%$ & 11.00 & $50 \%$ & $95 \%$ \\
(5) (2) and male urethral discharge & $67 \%$ & $17 \cdot 60$ & $40 \%$ & $98 \%$ \\
Predictor variables for Trichomonas vaginalis: & & & & \\
Clinics with or without pelvic exam capacity & & & & \\
(1) Vaginal discharge (any) & $18 \%$ & 1.32 & $71 \%$ & $46 \%$ \\
(2) (1) and female dysuria & $40 \%$ & 4.05 & $29 \%$ & $93 \%$ \\
(3) (1) and male dysuria & $35 \%$ & 3.27 & $50 \%$ & $85 \%$ \\
\hline
\end{tabular}

In Zambian health centres which cannot conduct pelvic examinations, treatment for gonorrhoea is based on a complaint of vaginal discharge. ${ }^{8}$ In our study, vaginal discharge was highly sensitive for gonorrhoea $(90 \%)$ but had a low predictive value $(16 \%)$ and likelihood ratio $(1 \cdot 72)$ (table). Thus, treatment based on a symptom of vaginal discharge would be inappropriate for most women. The addition of behavioural factors, such as a woman's alcohol use before sex or a husband's paying for sex, to vaginal discharge increases the algorithm's predictive ability. However, incorporating a husband's complaint of urethral discharge results in a highly discriminating algorithm (likelihood ratio $17 \cdot 6$ ) with adequate predictive value.

Management of gonorrhoea where pelvic examinations are conducted is based on clinical signs. ${ }^{8}$ A sign of yellow vaginal discharge alone had a low predictive value $(31 \%)$ and likelihood ratio $(4 \cdot 00)$. Adding either a woman's alcohol use before sex, a husband's paying for sex, or a symptom of urethral discharge to either yellow vaginal discharge or cervical erythema increased both predictive values (to $>56 \%$ ) and likelihood ratios (to $>11 \cdot 00)$.

Management of trichomoniasis in Zambia is also based on symptoms or signs of vaginal discharge. The positive predictive value and likelihood ratio associated with vaginal discharge alone for trichomoniasis is low (table). Algorithms incorporating female or male dysuria to vaginal discharge were more highly predictive.

\section{Discussion}

Based on the low prevalence of risky sexual behaviour among married African women, researchers have suggested that their STD risk is associated with their husbands' behaviour. ${ }^{67}$ Previous studies, however, were based on women's report of male behaviour. This study suggests that both female and male behaviour predict female STD in married Zambian women. For example, in multivariate analysis, a woman's alcohol use before sex and her husband's paying for sex were highly associated with gonorrhoea in women. These findings imply that prevention programmes should target both wives' and husbands' behaviour in high STD prevalence areas. Similar to previous findings from Africa, we found that the Zambian guidelines for syndromic management of non-ulcerative STD in women ${ }^{8}$ have low predictive value for $N$ gonorrhoeae and $T$ vaginalis. ${ }^{4910}$ Incorporation of women's sexual behaviour (numbers of sex partners) or sociodemographic factors (age, marital status) improved the predictive values of algorithms in some studies, ${ }^{5910}$ but not in others. ${ }^{4}$

We found that incorporating male and female sexual behaviour and male symptoms enhanced the predictive power of our algorithms. Improving such algorithms can enhance STD management thus reducing inappropriate treatment in resource-poor settings. These algorithms can also be used to 
improve women's utilisation of health care; a woman with vaginal discharge who discovers a partner's urethral discharge could be instructed to seek care immediately. They can also be used to counsel women at high STD risk about appropriate contraceptive options. However, the ability of a woman to give reliable information on her partner's symptoms and sexual behaviour should be verified.

Several limitations bear mentioning. Firstly, the small study size limits the precision of risk estimates and the scope of the multivariate analyses. Secondly, because the study population were clients who returned to the STD clinic with their spouses, it may not represent all married women attending the clinic. Our study population was similar in age, religion, and educational level with the general married population of Lusaka, however. ${ }^{11}$ Thirdly, the cross sectional nature of this analysis restricts our conclusions to correlates of prevalent rather than incident STD.

In summary, this study suggests that incorporating female and male sexual behaviour and male symptoms can improve diagnostic algorithms for STD among married African women. Efforts aimed at preventing STD in this population should target both husbands' and wives' behaviour.

Support for this study was provided by Family Health International (FHI) with funds from the US Agency for International Development (USAID). The views expressed in this article, however, do not necessarily reflect those of FHI or USAID. The authors wish to thank Dr Paul Feldblum, Dr Ward Cates, and Ms Carol Williamson for their review of this paper, Mr Rick McGirt for data entry and management, and the staff and clients of the Dermato-Venereology Clinic, University Teaching Hospital, Lusaka, Zambia, for their support and participation.

1 Wasserheit JN. Epidemiological synergy: interrelationships between human immunodeficiency virus infection and other sexually transmitted diseases. Sex Transm Dis 1992;19:61-77.

2 Laga M, Manoka A, Kivuvu M, Marele B, Tuliza M, Nzila $\mathrm{N}$, et al. Non-ulcerative sexually transmitted diseases as risk factors for HIV-1 transmission in women: results from a cohort study. AIDS 1993;7:95-102.

3 Hunter DJ, Maggwa BN, Mati JKG, Tukei PM, Mbugua $S$. Sexual behavior, sexually transmitted diseases, male circumcision and risk of HIV infection among women in Nairobi, Kenya. AIDS 1994;8:93-9.

4 Daly CC, Maggwa N, Mati JK, Solomon M, Mbugua S, Tukei PM, et al. Risk factors for gonorrhoea, syphilis, and trichomonas infections among women attending family planning clinics in Nairobi, Kenya. Genitourin Med 1994;70:155-61.

5 Mayaud P, Grosskurth H, Changalucha J, Todd J, West B, Gabone $\mathbf{R}$, et al. Risk assessment and other screening options for gonorrhoea and chlamydial infections in women attending rural Tanzanian antenatal clinics. Bull WHO 1995;73:621-30.

6 Allen S, Lindan C, Serufilira A, Van der Perre P, Rundle $A C$, Nsengumuremyi $F$, et al. Human immunodeficiency virus infection in urban Rwanda: demographic and behavioral correlates in a representative sample of childbearing women. $\mathscr{f} A M A$ 1991;266:1657-63.

7 Hunter DJ. AIDS in sub-Saharan Africa: the epidemiology of heterosexual transmission and the prospects for prevention. Epidemiology 1993;4:63-71.

8 National STD Control Programme. Sexually transmitted diseases (STD): guidelines for diagnosis and treatment. Lusaka, Zambia: Ministry of Health, 1991

9 Braddick MR, Ndinya-Achola JO, Mirza NB, Plummer FA, Irungu G, Sinei SK, et al. Towards developing a diagnostic algorithm for Chlamydia trachomatis and Neisseria gonorrhoeae cervicitis in pregnancy. Genitourin Med 1990;66:62-5.

10 Vuylsteke B, Laga M, Alary M, Gerniers MM, Lebughe JP, Nzila $\mathrm{N}$, et al. Clinical algorithms for the screening of women for gonococcal and chlamydial infection: evaluation of pregnant women and prostitutes in Zaire. Clin Infect Dis 1993;17:82-8.

11 University of Zambia, Central Statistical Office, Ministry of Health (Government of Zambia). Zambia Demographic and Health Survey 1992. Columbia, MD: Macro International, 1993. 